\title{
Locating Error when Clamping Workpiece between Centers
}

\author{
Laurentiu Slătineanu ${ }^{1}$, Margareta Coteaţă ${ }^{1}$, Oana Dodun ${ }^{1}$, Gheorghe Nagîţ ${ }^{1}$, Adelina \\ Hriţuc $^{1, *}$, Irina Beşliu-Băncescu ${ }^{2}$, and Mihai Boca ${ }^{1}$ \\ 1"Gheorghe Asachi” Technical University of Iaşi, Department of Machine Manufacturing \\ Technology, Blvd. D. Mangeron, 59A, 700050 Iaşi, România \\ 2"Ştefan cel Mare" University of Suceava, Department of Mechanics and Technologies, Universităţii \\ Street 13, Suceava 720229, România
}

\begin{abstract}
The total machining error includes some distinct errors summed up by following certain rules. One of these errors is the workpiece locating error, which occurs when the workpiece reference base does not match the workpiece locating surface. The paper analyses different definitions of positioning error, as they were proposed by different researchers or groups of researchers and presents an application valid in the case of turning a cylindrical workpiece with locating between the centers. A definition of locating error that takes into account the total differential of the size of the vector that joins the orientation surface with an element of the fixture was preferred. A fixture version was considered to ensure the locating and clamping of the workpiece on the lathes, with workpiece locating between the centers. This fixture uses a mandrel with a sliding center and an elastic collet.
\end{abstract}

\section{Introduction}

Machining accuracy is a concept that refers to the correspondence that exists between the values of some parameters that define the dimensions, shape and position of some surfaces and the values of the same parameters inscribed in the mechanical drawing of a part [1]. In general, the machining accuracy can be evaluated by the so-called machining error, this being the difference between the nominal value of the accuracy parameter used and the actual value of the same parameter.

There are a large number of factors that can influence the machining accuracy. For this reason, the component of the total machining error includes the errors determined by the wear, thermal and elastic deformation of the components of the machining system, by the geometric deviations of the surfaces of some components of the machining system, by the setting of the processing system, etc. Given that such errors are usually vector sizes, their summation to estimate the value of the total machining error is performed according to the rule of square root, but also intervening dispersion coefficients that take into account the statistical distribution of the values of certain components of the total machining error.

\footnotetext{
*Corresponding author: hrituc.adelina3295@yahoo.com
} 
One of the components of the total machining error is the locating error of the workpiece on the table of the machine tool or in a fixture intended even for locating and clamping the workpiece to develop to machining process that generates machining forces. In principle, it is considered that the locating error occurs when we are dealing with a machining process that uses tools whose cutting tips are properly adjusted to obtain certain dimensions of the surfaces to be machined. The locating error is assessed as a difference between the positions of the so-called technological measuring surfaces, when the measuring surfaces do not coincide with the locating surfaces.

Given the importance of the locating error for obtaining precisely the various surfaces of the part, it was normal for researchers to investigate how the locating error occurs and how to reduce or even cancel its effects.

Thus, Zhang et al analyzed the propagation of the workpiece locating error when using multistation manufacturing systems [2]. They developed a mathematical model that takes into account the variation of some of the factors used to calculate the locating error.

Khodaygan considered that the locating error exerts a significant influence on the assemblability and final quality of a product [3]. He proposed a mathematical model to highlight the influence of different factors on the size of the locating error. In this model, the dimensional and geometric errors, dimensions and tolerances of certain dimensions of the workpieces were taken into account, as well as the results of some statistical approaches.

Parvaz et al. they developed a theoretical model for the analysis of the locating errors of the workpiece and respectively a computer-aided assembly model for the calculation of the locating error [4]. Performing some experimental tests confirmed the validity of the theoretical predictions.

Tang et al. developed an analysis of the workpiece locating error taking into account general fixture layouts and parameterized tolerances [5]. They proposed two mathematical relations to calculate the errors in the locating points for two distinct situations.

The paper addressed the issue of determining the locating error when clamping workpieces between centers to a lathe machining. To cancel the locating error and thus increase the machining accuracy, a device that uses the locating between the centers and an elastic collet was proposed.

\section{Theoretical modelling of the locating error when clamping workpiece between centers}

The locating error is a component of the workpiece set up error, along with the clamping error and the error of the fixture used to locate and clamp the workpiece.

There are different ways to interpret the meaning and calculate the locating error. According to its definition, the error is due to the inconsistency of the reference (measurement) base with the locating surface and is given by the projection of the displacement of the reference (measurement) base in the direction of the size to be obtained by machining [6].

Another definition of locating error, which uses differential calculation elements, specifies that the locating error can be calculated using the total differential of the expression corresponding to the projection of a vector connecting the workpiece locating elements (for example, workpiece locating surface) to the locating elements. of the fixture used to locate and clamp the workpiece.

We will consider the case of workpiece located between the centers when rotating the workpiece using a lathe dog. We will assume that we are dealing with a machining of a number of workpieces on a technological system with the adjustment of the dimensions to be obtained (for example, by means of stops). In this way, the cylindrical surface of length $L_{1}$ is 
machined (Fig. 1), without adjusting the cutting tool position to the dimension to be obtained of each workpiece.

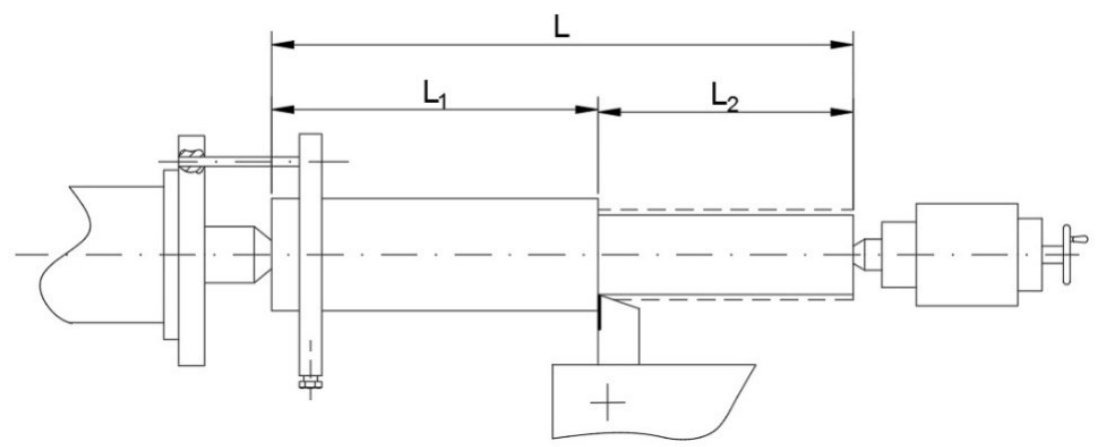

Fig. 1. Turning an outer cylindrical surface with a tool adjusted to the length of the cylindrical zone to be obtained, in case of a series production.

If it is necessary to obtain the $L_{1}$ dimension with high accuracy, we will find that a locating error $\varepsilon_{I L 1}$ occurs, the size of which will be essentially affected by the variation of the depth of the centering hole (previously made considering a certain tolerance for the hole depth). Since it is easier to practically determine the diameter $D_{c h}$ of the centering hole at the left front surface of the workpiece, instead of the depth of the centering hole, the diameter $D_{c h}$ will be taken into account and we will find a $\Delta D_{c h}$ dispersion of this diameter [1]:

$$
\Delta D_{\text {ch }}=D_{\text {ch } \max }-D_{\text {ch } \min }
$$

To use that definition of the locating error based on the use of the total differential, we will consider as the locating element of the device the theoretical tip of the cone corresponding to the fix center mounted in the main shaft of the lathe (Fig. 2). As locating element of the workpiece, we will take into consideration the centering hole surface from the left zone of the workpiece. This is how we reach the right triangle BAD, where the following relation can be written:

$$
\operatorname{tg} \alpha=\frac{\Delta D_{c h}}{2 l}
$$

where $\alpha$ is the angle at the top of the cone, and $l$ is the length of the segment AB, corresponding to the size of the vector mentioned in the definition of the orientation error.

We can therefore write as an expression of the size $l$ of this vector:

$$
l=\frac{\Delta D_{c h}}{2 \operatorname{tg} \propto}
$$

In principle, the total differential of this quantity will be given by the mathematical relation:

$$
\varepsilon_{l L 1}=\Delta l=\frac{\partial l}{D_{c h}} \Delta D_{c h}+\frac{\partial l}{\partial \alpha} \Delta \alpha
$$

A general expression of the locating error in this case, according to [1] has the form:

$$
\varepsilon_{l L 1}=\sqrt{k_{1}^{2}\left(\frac{\partial l}{\partial D_{c h}}\right)^{2} \Delta D_{c h}^{2}+k_{2}^{2}\left(\frac{\partial l}{\partial \alpha}\right)^{2} \Delta \alpha^{2}},
$$


where $k_{1}$ and $k_{2}$ are relative dispersion coefficients.

Considering the relation (3), the locating error in its general form will be therefore:

$$
\varepsilon_{l L 1}=\frac{1}{2 \operatorname{tg} \alpha} \Delta D_{c h}+\frac{D_{c h}}{2} \cdot \frac{1}{\cos \alpha} \Delta \alpha
$$

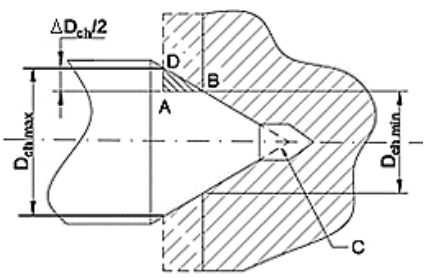

Fig. 2. Displacement of the measuring base due to the variation of the outer diameter of the centering hole.

Assuming that we are not dealing with a variation of the $\Delta \alpha$ size of the $\alpha$ angle (which means $\Delta \alpha=0$ ), we arrive at the particular case:

$$
\varepsilon_{l L 1}=\frac{\Delta D_{c h}}{2 \operatorname{tg} \alpha}
$$

Such a relationship, which corresponds, as mentioned, to a particular case of the locating error, is also found in the literature $[1,6,7]$.

\section{Fixture using the live center}

In accordance with the abovementioned aspects, the coincidence of the locating surface with the reference base specifying the position of a machined surface provides conditions for cancelling the locating error and increasing, as such, the machining accuracy when applying a technology that uses tools adjusted to the length of the surface to be machined.

At present, fixtures are used for machining between the centers, using a sliding fix center which moves together with the workpiece, when acting on the handwheel for the axial movement of the tailstock quill. This movement takes place until the left front surface of the workpiece comes into contact with some support parts of the device (multicomponent fix center), parts which will no longer allow a leftward movement of the workpiece. The support parts are, in fact, provided with sharp edges, intended to allow penetration by elastic deformation of the material corresponding to the left front surface of the workpiece and thus to transmit the torque necessary to drive the rotating motion of the workpiece during machining process.

As the transmitted torque cannot be too high, such fixtures (multicomponent live centers) are used more in grinding or finishing turning and less in roughing turning, when the higher torque could cause either damage of left front surface of the workpiece, or simply sliding the support parts onto this left front surface of the workpiece. 
To reduce the aforementioned disadvantage, a solution was initially proposed [8] to ensure an initial locating of the workpiece between the centers, so that later, by moving the sliding center, to ensure the clamping of the workpiece in an elastic collet.

In this way, it became possible to transmit a higher torque than when using sharp-edged

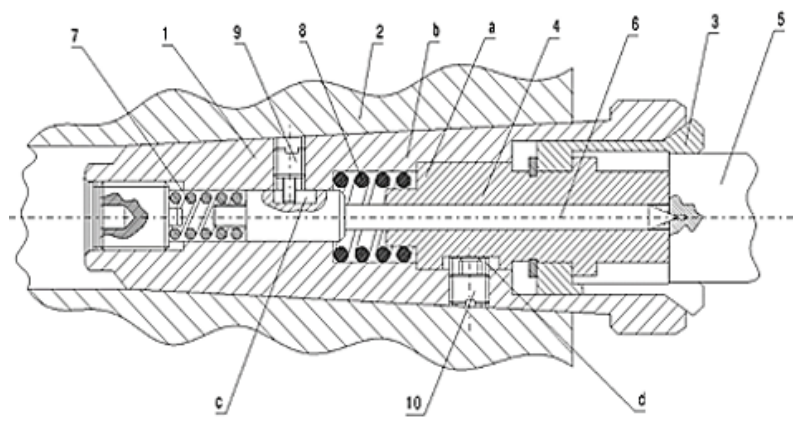

Fig. 3. Mandrel with elastic collet designed to ensure the cancellation of the locating error and the rotational drive of the workpiece: 1 - mandrel body; 2 - lathe main shaft; 3 - elastic collet; 4 bushing with stepped zones; 5 - workpiece; 6 - sliding center; 7, 8 - springs; 9,10 - threaded bolts.

support parts. However, such a solution did not allow the locating error to be cancelled.

It was appreciated that it is possible to improve this situation using the solution shown in Figure 3. In this case, it can be seen the use of the known spring loaded sliding center solution, whose displacement causes compression of a spring that will allow subsequent return to the right of the sliding center, when removing the previously machined workpiece.

In the case of the proposed solution, after locating the workpiece on the sliding center on the left and moving the workpiece to the left, by turning the hand wheel on the lathe tailstock, we will reach the situation where the left front surface of the workpiece contacts the right front surface of bushing 4 with stepped zones on the outside. Further rotation of the handwheel from the lathe tailstock will cause the bushing 4 to move to the left and the spring 8 to be compressed.

Moving the bushing 4 to the left will be possible until the shoulder $a$ of the bushing 4 contacts the shoulder $b$ in the bore of the conical body 1 of the fixture. At the same time, the movement to the left of the bushing 4 will determine the clamping of the workpiece 5 by the elastic collet 3 .

It is obvious that the axial movements of the sliding center 6 and the sleeve 4 must be limited, in order to avoid their exit outside the body 1 of the fixture, under the action of the expansion of the springs 7 and 8 .

Threaded bolts 9 and 10 have been provided as such their ends move in some channels located in the sliding center 6 and in the bushing 4, respectively.

The constructive solution described above requires a high machining accuracy of certain surfaces of the components of the fixture (mandrel with elastic collet), to ensure the adequate clamping of the workpiece 5 in the elastic collet 3 , when the shoulder $a$ of the bushing 4 contacts the shoulder $b$ of the bore of the mandrel body 1 . 


\section{Conclusions}

The examination of the components of the total machining error highlighted the existence of some possibilities to diminish this total machining error by cancelling the workpiece locating error to the machining between the centers. It has been found that there are several ways to define and calculate the locating error. In this paper, a definition version based on differential calculation was preferred. This way of evaluating the size of the locating error was used to arrive at general expressions corresponding to the locating error when locating the workpieces between the centers on lathes or on grinding machines when machining external cylindrical surfaces. Attempts to outline a fixture solution that would allow both the cancellation of the locating error and the provision of conditions for the rotational drive of the workpiece led to the design of a mandrel-type device with an elastic collet. In the future, it is intended to materialize this solution and experimental verification of how it ensures the fulfilment of the conditions of cancellation of the locating error and respectively of adequate training of the workpiece in rotating motion.

\section{References}

1. C. Picoş, Machine manufacturing technology (in Romanian) (Editura Didactică și Pedagogică, Bucharest, 1974)

2. F. Zhang, J. Lu, S. Tang, H. Sun, L. Jiao, Chin. J. Mech. Eng. 23, 765 (2010)

3. S. Khodaygan, SAE Technical Paper 1, 1347 (2016)

4. H. Parvaz, M. Bodaghy Aleny, AUT J. Mech. Eng. 4, 229 (2020)

5. W. Tang, Y. Li, J. Yu, J. Zhang, L. Yu, Proc. IMechE Part B: J. Engg. Manuf. 230, 416 (2014)

6. N. Gojinețchi, Device design course. First Part (in Romanian) (Institutul Politehnic, Iași, 1970)

7. N.K. Mehta, Metal cutting and design of cutting tools, jigs \& fixtures (McGraw Hill Education Private Limited, 2015)

8. E. Colceriu, L. Slătineanu, T. Grămescu, Patent Romania RO86708B1 (1983) 